\title{
Signal Analysis of Broken Wires Fault with Different Width
}

\author{
Guohua Gao a, Lin Gao b \\ College of Mechanical Engineering and Applied Electronics Technology, Beijing University of \\ Technology, Beijing, 100022, China \\ aggh0912@126.com, b378118307@qq.com
}

Keywords: Wire rope, MFL, Broken wires, Fault width.

\begin{abstract}
To guarantee personal and property security, the operational status of wire ropes need to real time inspection.in this study, the static magnetic field of wire rope with different broken wires width was analyzed and calculated using finite element method, and the software named Ansoft Maxwell is used to model and simulate as a tool, in experiment, the magnetic flux leakage (MFL) method is used to detect the typical defects, wavelet transform was used to eliminate background noise and to detect singularity. Wave width and peak-to-peak value as characteristic index to measure the fault signal. Simulation results indicate the leakage magnetic field components has showed growth trend with increasing fault width, Similarly, the experiment results show that the peak-to-peak values and wave width of the MFL signal increase and peak-to-peak value increases quickly compared with wave width. The results of this study have significant implications for quantitative measurement of broken wires defect detection of wire rope.
\end{abstract}

\section{Introduction}

With the rapid economic development, wire ropes, as a key component of pulling, got a wide application in ports, lifting, coal, elevators, etc. However, it is unavoidable that wire ropes will produce wear, broken wire and other damages because of long-term use, if the existing problems cannot be discovered and solved, it threatens the public security and people s safety and healthy. Currently, many departments and enterprises mainly use artificial detection method to judge the damage, but it cannot directly observe the internal damage, which can cause a lot of time and personnel waste [1-3].

Among the various detection methods of wire ropes, magnetic flux leakage (MFL) method is considered to be the most reliable detection because of good quality and high reliability. The principle of the magnetic flux leakage (MFL) detection is the change of the leakage magnetic field on the surface of wire ropes. Generally, wire ropes are magnetized by permanent magnets in the first stage then, magneto-dependent sensors, such as inducting coils or Hall sensors are used to detect the faults of wire ropes [4].

With the development of testing technology, more and more research has been done on the fault of wire ropes. In 2013, a radial basis function neural network model used for the quantitative recognition of wire ropes fracture damage was designed by Liu Xiaoping in Beijing University of Posts and Telecommunications, but those research focused on the number of broken wires rather than the width, the study of broken wires fault with different width is rare [5].

In this paper, we study the in detail the static magnetic field of wire rope with different broken wires width using Ansoft Maxwell, at the same time, we produced the broken wires fault and conducted the experiment. Wave width and peak-to-peak value as characteristic index were selected to measure the fault signal. In order to ensure validity of conclusion, the experimental results will be compared with the simulation results. 


\section{Maxwell}

In this section, the static magnetic field of the excitation system magnetizing wire rope was analyzed and calculated using finite element method. The software named Ansoft Maxwell is used to model and simulate as a tool.

The excitation system is composed of magnet,armature and wire rope. Considering the experiment in practice, the 3-D models of the excitation device and wire rope ,as presented in Figure 3, were built in Maxwell three-dimensional (3D) software, as shown in Figure 1. In this model, M54 steel was used as a simulation model of wire rope whose diameter is $12.5 \mathrm{~mm}$. NdFe35 is selected for the permanent magnet material, the sizes of magnet and armature are same: inside diameter is $22.5 \mathrm{~mm}$, outside diameter is $52 \mathrm{~mm}$, thickness is $10 \mathrm{~mm}$. The physical parameters of broken wire defects was defined as figure, $h$ is the depth of the fault, $b$ is the width of the fault, $l$ is the length of the fault.

After setting the definition of material and boundary conditions and grid subdivision, the model based on the method of the static magnetic field simulation analysis was calculated by Maxwell. The magnetic strength distribution nephogram of broken wire defect observed from Fig.2. which shows that the influence of the physical parameter 1 on the signals of magnetic flux leakage can be obtained through examining the leakage magnetic field on the surface of broken wires.

In Maxwell simulation, the length of the faults is divided into five levels and gradually increased from $0 \mathrm{~mm}$ to $10 \mathrm{~mm}$ at a tolerance $2 \mathrm{~m}$. the depth and width of the fault are kept at $3 \mathrm{~mm}$. the radial components of leakage magnetic field were extracted at the coordinate point (0.3.0) .

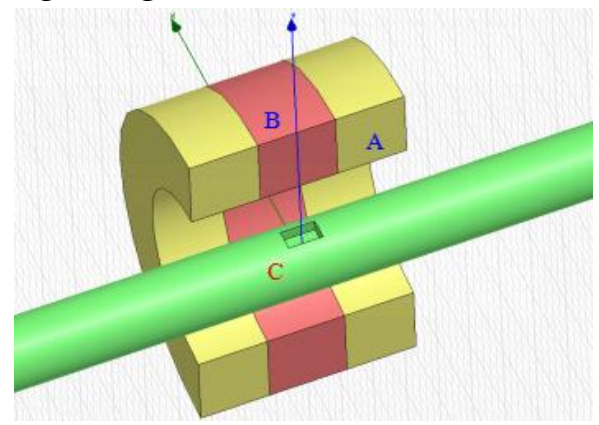

a) Simulation model

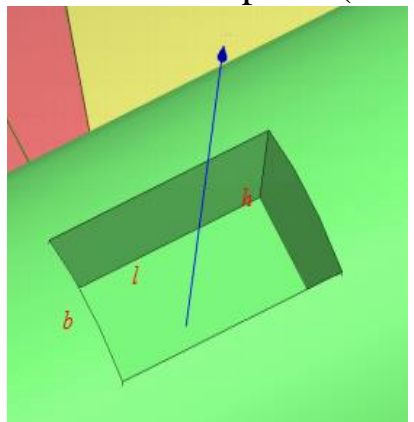

b) Defect parameters

A: Magnet; B: Armature; C: Broken wires; h: Fault depth; 1: Fault length; b: Fault width

Fig. 1 The 3D simulation model of magnetic flux leakage testing

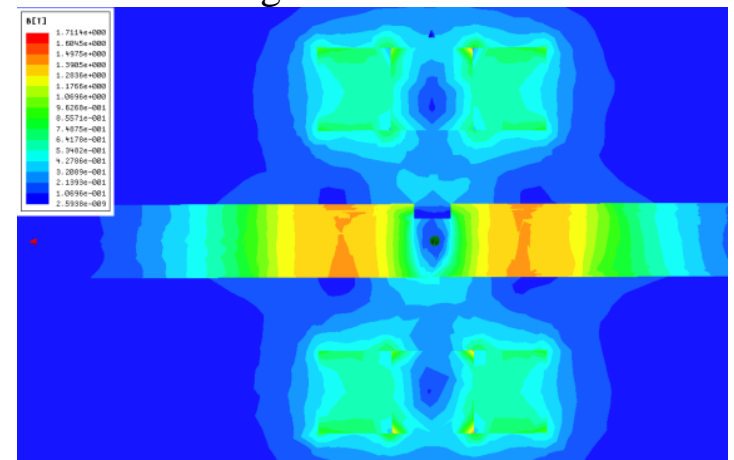

a) X-Y plane

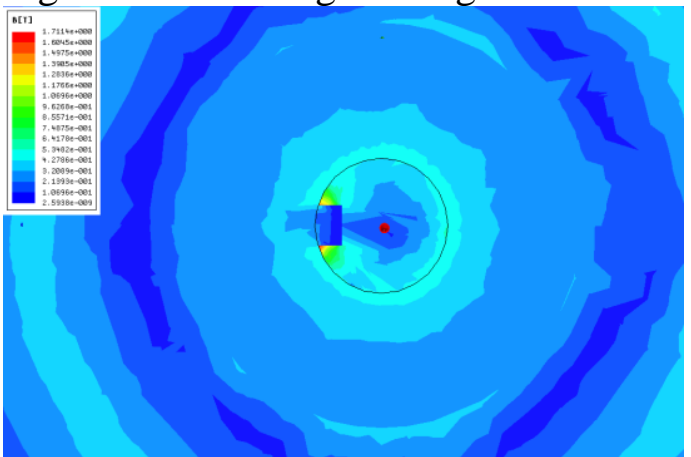

b) X-Z plane

Fig. 2 The magnetic strength distribution nephogram of broken wire defects

\section{Reproducing of sample wire ropes and experiment platform}

The nominal diameter of wire ropes in regular lay selected in experiments is $\Phi 12.5 \mathrm{~mm}$, their structure specification is $6 \times 19+\mathrm{FC}$ and the diameter of the outer strand wires is $\Phi 0.8 \mathrm{~mm}$. The calculated sectional area of samples is $52.27 \mathrm{~mm} 2$. The nominal anti-tensile strength is $1770 \mathrm{MPa}$.

As the detected objects steel wire ropes with are produced. In this study, the damage degree of broken wires is made relatively severe, which is helpful to preferably extract the amplitude of the magnetic signal at the defect point. Based on the ASTM E1507-2001, the different fault interval 
lengths of broken wire are selected and produced. Sections of the defect samples are shown in Figure 3. The detailed fault information is listed in Table 1 .

Table 1. Information on broken wires faults

\begin{tabular}{|c|c|c|c|}
\hline Fault type & Wire rope number & $\begin{array}{c}\text { Fault degree } \\
\text { (Breaking rate) }\end{array}$ & $\begin{array}{l}\text { Damaged interval length } \\
(\mathrm{mm})\end{array}$ \\
\hline \multirow{6}{*}{ Broken wires } & No. 1 & \multirow{6}{*}{$\begin{array}{c}5 \text { broken wires } \\
(4.39 \%)\end{array}$} & 0.5 \\
\hline & No. 2 & & 2 \\
\hline & No.3 & & 4 \\
\hline & No.4 & & 6 \\
\hline & No.5 & & 8 \\
\hline & No.6 & & 10 \\
\hline
\end{tabular}

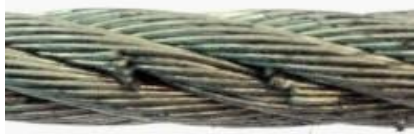

a) $10 \mathrm{~mm}$

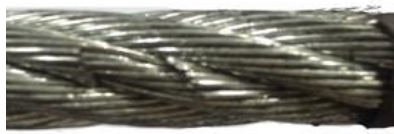

b) $8 \mathrm{~mm}$

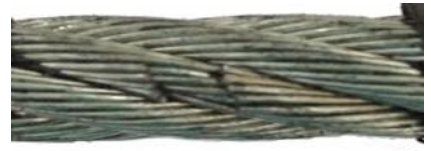

c) $6 \mathrm{~mm}$

Fig.3 Section of broken wires faults

\section{Experiment and result}

The broken wire sample wire rope run on the experimental platform through a wheel train structure, and was applied lateral variable load by a hydraulic oil cylinder. The running speed was kept at 1 meter per second. The wire rope went through a MFL sensor while running, then the sensor collected the signals of wire rope and transmitted it to the PC for processing, as shown in figure 4.

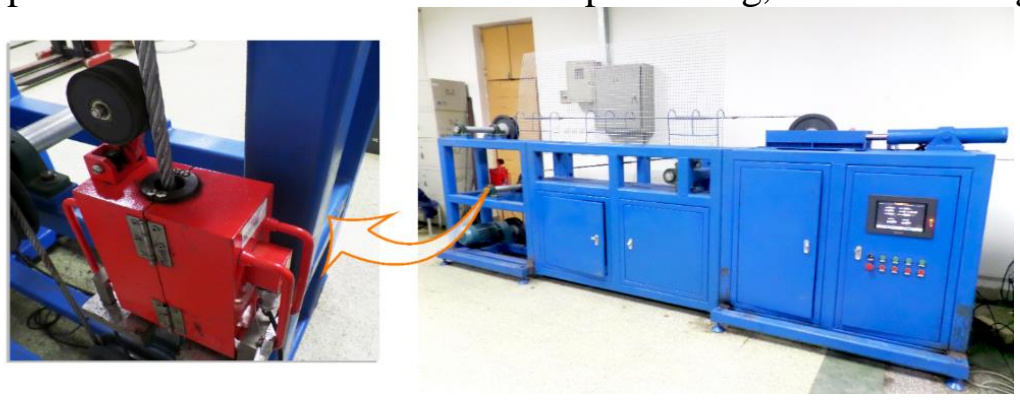

Fig. 4 The detection platform

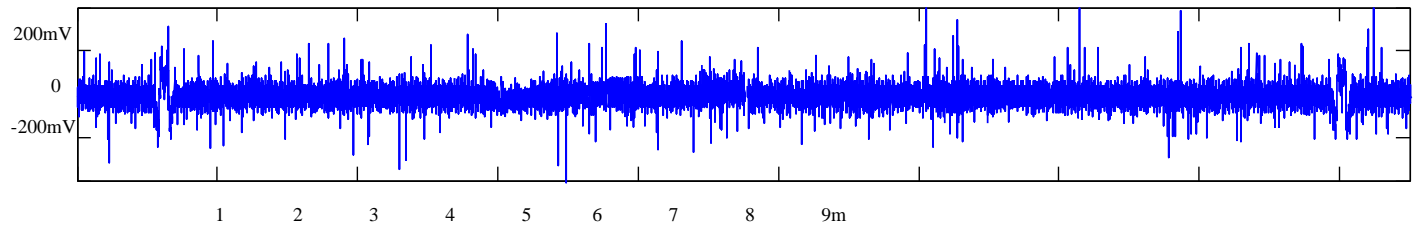

a) the original signal

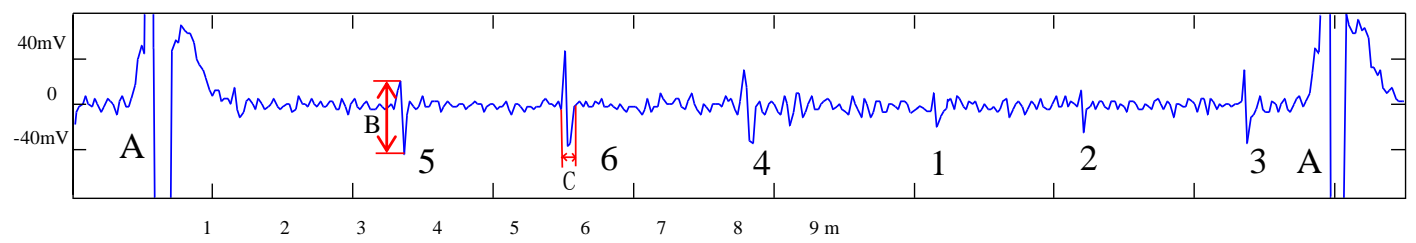

b) the wavelet reconstruction signal

A: The rope connector; B: peak-to-peak value; C: waviness width $1,2,3,4,5,6$ : broken wiress with the width from $0.5 \mathrm{~mm}$ to $10 \mathrm{~mm}$

Fig.5 Fault Signal

The MFL signal of wire rope is a kind of low frequency signal and itsfrequency range of $100 \mathrm{~Hz}$ to $150 \mathrm{~Hz}$. Therefore the low frequency component is our main object to analyze. In this paper, we chose $\mathrm{Db} 4$ wavelet to possess an optimum decomposition and reconstruction ability. The original 
signal is collected from the sample wire ropes with broken wire defects, and then decomposed into 7 layers by using Db4 wavelet. The coefficients of high frequency in each scale are set to zero, and the low frequency components of 7 th layers are used to reconstruct the signals.the signals as shown in figure 5 .

In each particular experiment, we extracted the peak-to-peak value and waviness width of fault point, which is used to measure the leakage magnetic field, we define characteristic values as shown in Figure5(b). in order to eliminate the effects of accidental errors, the average of these values were measured 20 times continuously. Each average peak-to-peak value and waviness width of the three and five broken wires defect point is listed in Tables 2. The simulation result By and real experiments shows that the vertical components of leakage magnetic field, peak-to-peak value and waviness width has showed growth trend with the increase of fault width, peak-to-peak value.

Table 2. The result of simulation and real experiment

\begin{tabular}{|c|c|c|c|c|c|c|}
\hline Broken wires width (mm) & 0.5 & 2 & 4 & 6 & 8 & 10 \\
\hline By (T) & 0.021 & 0.062 & 0.073 & 0.088 & 0.103 & 0.115 \\
\hline peak-to-peak value(mv) & 21.02 & 29.35 & 45.19 & 59.21 & 74.53 & 85.32 \\
\hline waviness width(ms) & 18.09 & 25.32 & 30.32 & 34.44 & 38.52 & 42.56 \\
\hline
\end{tabular}

\section{Conclusion}

In this paper, MFL was used to detect the broken wires fault, the static magnetic field of the excitation system magnetizing wire rope was analyzed and calculated using finite element method. In experiment, Sample wire ropes with different width broken wires was reproduced by plier, the background noise of the original signals can be eliminated effectively through wavelet decomposition and reconstruction. Wave width and peak-to-peak value as characteristic index to measure the fault. The result shows that the components of leakage magnetic field has showed growth trend with the increase of fault width. Similarly, wave width and peak-to-peak value indicate the same growing trend which showed the results of simulation agree well with the experimental data. Furthermore, peak-to-peak value increases quickly compared with wave width. The thorough study of the signal with different width faults is very meaningful for quantitative measurement of the broken wires.

\section{References}

[1]. Li YF. Study of the measurement of the effect of coal resource development on resource environment in mining area. Journal of China University of Mining \& Technology Vol. 39 (2009) No. 4; p. 607-608

[2]. Zhong X Y, Zhang X H. 'Research of Non-Destructive Testing of Wire Rope Using Magnetic Flux Leakage', Applied Mechanics and Materials, Vol. 189(2012), p.255-259.

[3]. Jianxin C, Wei G. 'Study and development on detecting device of wire rope localized fault', Proceedings of the 4th World Congress on. Shanghai, 2002.6, IEEE, p. 704-707.

[4]. Park S H, Kim J W, Nam M J, et al. 'Magnetic flux leakage sensing-based steel cable NDE technique incorporated on a cable climbing robot for bridge structures', Advances in Science and Technology, Vol. 83(2013), p.217-222.

[5]. Chen $j$. The research on wire rope quantitative detection and safety evaluation [Mater Dissertation], Beijing University of Posts and Telecommunications, 2013, p10 